\title{
The stringy quantum Hall fluid
}

\section{Oren Bergman and Yuji Okawa}

California Institute of Technology

Pasadena CA 91125, USA and

CIT/USC Center for Theoretical Physics, Univ. of Southern California

Los Angeles CA, USA

E-mail: 'bergman@theory.caltech.edú, lokawa@theory.caìtech.edu'

\section{John Brodie}

Stanford Linear Accelerator Center, Stanford University

Stanford, CA 94305, USA

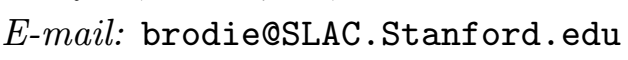

ABstract: Using branes in massive type IIA string theory, and a novel decoupling limit, we provide an explicit correspondence between non-commutative Chern-Simons theory and the fractional quantum Hall fluid. The role of the electrons is played by D-particles, the background magnetic field corresponds to a RR 2-form flux, and the two-dimensional fluid is described by non-commutative D2-branes. The filling fraction is given by the ratio of the number of D2-branes and the number of D8-branes, and therefore by the ratio rank/level of the Chern-Simons gauge theory. Quasiparticles and quasiholes are realized as endpoints of fundamental strings on the D2-branes, and are found to possess fractional D-particle charges and fractional statistics.

Keywords: iChern-Simons Theories, D-branes'. 


\section{Contents}

i.: Introduction

2. The non-commutative massive D2-brane as a QH fluid

'2.1' D2-brane in massive IIA supergravity

12.2 Non-commutative D2-brane

Quantum Hall fluid

2.- Multiple D2-branes

3. D-particles and the Chern-Simons matrix model

'i.j.' D-particles in massive IIA supergravity

13.2 The Chern-Simons matrix model

13.3\% Solutions

4. Quasiparticles and quasiholes

'Ā.ì' Space-time picture

Matrix model picture

Charge distribution

5. Conclusions and outlook

\section{Introduction}

Many physical systems can be described, at least in some dynamical regime, by gauge field theories. Indeed, gauge field theories are considered fundamental in understanding the interactions of elementary particles. In recent years, gauge theories have also been applied to the study of low-energy systems in condensed matter physics [i]1] such as the quantum Hall fluid [2], and high- $\mathrm{T}_{c}$ superconductors [3기. In this case gauge theory provides an effective, rather than fundamental, description, which still proves to be quite useful. In both cases, a better understanding of the dynamics of gauge field theories is clearly a worthwhile pursuit.

It has recently been conjectured by Susskind that a two-dimensional quantum Hall fluid of charged particles with filling fraction $\nu=1 / k$ is described by a noncommutative Chern-Simons (NCCS) gauge theory at level $k$ [4]. This conjecture is based on an earlier realization that a two-dimensional charged fluid in a strong magnetic field is equivalent to a pure Chern-Simons (CS) gauge theory, where the 
gauge group is the group of area-preserving diffeomorphisms (APD) [5usskind argued that in order to fully realize the graininess of the fluid one must replace the above CS theory with a non-commutative U(1) CS theory. The APD CS theory can be thought of as the lowest order approximation to the NCCS theory. Some evidence for this conjecture was provided in [6], where a formal correspondence between the Hilbert space of NCCS theory and Laughlin's wavefunctions [i] was shown. (For a more detailed discussion of the correspondence see [8] $\left[\begin{array}{l}8] \\ 1\end{array}\right)$.

Studies of string dualities have led to novel realizations of various gauge field theories using configurations of branes, and much has been learned about the dynamics of gauge theories from these configurations $\left[\begin{array}{l}\overline{9} \\ \mathbf{n}\end{array}\right]$. Of course it is not at all clear that nature chooses to realize gauge field theories in this way. Nevertheless, it is useful to study a variety of realizations of gauge theories. In particular, certain realizations may provide insights into a dynamical regime of the theory where other realizations, perhaps even the true realization, are not as useful. It is therefore an interesting question whether NCCS theory can be realized on branes in string theory.

It is well known that D-branes in a constant $B$ field background, and in a certain zero-slope limit known as the Seiberg-Witten (SW) limit, give rise to noncommutative gauge theories [i-10 ind ${ }^{1}$ On the other hand, it is also known that the world-volume theories of D-branes in massive Type IIA string theory [i] 3 , i.e. in the presence of D8-branes, contain CS terms [1, 14$]$. In particular, the theory on a D2-brane near D8-branes is three-dimensional Yang-Mills-Chern-Simons (YMCS) theory, with some scalars and fermions. It is therefore natural to look for a brane realization of NCCS theory using D2-branes in massive IIA string theory with a $B$ field, and this is indeed where we will find it. Furthermore, we will show that a consistent massive IIA background with non-vanishing $B$ requires a non-vanishing $\mathrm{RR}$ magnetic field $G_{2}$ as well. Since $B$ also induces a uniform D-particle density in the D2-brane, the entire system is found to correspond to a two-dimensional fluid (the D2-brane) of charged particles (the D-particles) moving in a uniform magnetic field $\left(G_{2}\right)$, namely a quantum Hall fluid. Thus the brane picture draws a correspondence between NCCS theory and the quantum Hall fluid.

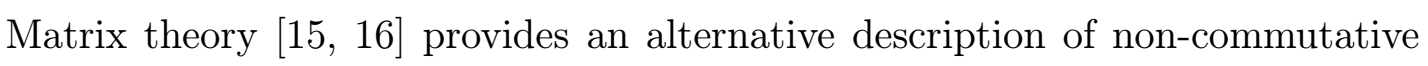
gauge theory in terms of lower-dimensional D-branes. For example, a non-commutative configuration of two matrices $X^{1}, X^{2}$ in the matrix model corresponds to a D2brane with a uniform density of D-particles. Fluctuations about this configuration are then governed by a non-commutative SYM theory [i] be derived from a certain matrix model [1] 18. with the electrons of the quantum Hall fluid [i] can also be realized in terms of D-particles. In an appropriate decoupling limit, the dynamics of D-particles in massive IIA string theory with a $B$ field are governed

\footnotetext{
${ }^{1}$ See also a recent review [1] $\left.{ }^{1}\right]^{\eta}$.
} 
by a pure CS matrix model, which in turn reproduces the three-dimensional NCCS theory on the D2-brane.

Strings play a central role in the D-particle matrix model. Their presence on D-particles in massive IIA string theory is a known consequence of charge conservation [25, 126$]$, which in the matrix model formulation is encoded in the Gauss law constraint. The latter exhibits a variety of solutions, including a non-commutative D2-brane with no strings. Solutions containing both a D2-brane and strings ending on it are identified with quasiparticles and quasiholes in the quantum Hall fluid. These are found to carry fractional D-particle charge, and to exhibit fractional statistics.

The paper is organized as follows. In section we show that the world-volume theory on D2-branes in massive IIA string theory with a constant $B$ field background reduces to pure NCCS theory in an appropriate decoupling limit. This gives a derivation of the conjectured correspondence of NCCS theory and the quantum Hall fluid. In section $\overline{3}_{1}$ we describe the matrix model of D-particles in massive IIA string theory, which gives rise to NCCS theory on the D2-brane. In section 集we identify the quasiparticles and quasiholes of the quantum Hall fluid, in both the spacetime picture and the matrix model description, and determine their charge and statistics. Section is devoted to our conclusions and outlook.

Note added: While this paper was being finished, we were informed of work by L. Susskind and S. Hellerman on a string theory construction of the quantum Hall system [i20].

\section{The non-commutative massive D2-brane as a $\mathrm{QH}$ fluid}

\subsection{D2-brane in massive IIA supergravity}

Our starting point is massive type IIA supergravity [1] $\left.\overline{3}_{1}\right]$. In particular, we shall be interested in a form of the (bosonic) action which includes the 9-form potential

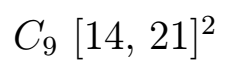

$$
\begin{aligned}
S= & \frac{1}{2 \kappa_{10}^{2}} \int d^{10} x \sqrt{-g}\left\{e^{-2 \Phi}\left[R+4|d \Phi|^{2}-\frac{1}{2}|H|^{2}\right]-\frac{1}{2}\left|\widetilde{G}_{2}\right|^{2}-\frac{1}{2}\left|\widetilde{G}_{4}\right|^{2}-\frac{1}{2} M^{2}\right\}- \\
& -\frac{1}{4 \kappa_{10}^{2}} \int\left\{2 M d C_{9}+B \wedge\left(d C_{3}+\frac{1}{2} M B \wedge B\right) \wedge\left(d C_{3}+\frac{1}{2} M B \wedge B\right)\right\},(2.1)
\end{aligned}
$$

where $\kappa_{10}^{2}=(2 \pi)^{7}\left(\alpha^{\prime}\right)^{4} / 2$, and the gauge-invariant field strengths are given by

$$
\begin{aligned}
H & =d B, \\
\widetilde{G}_{2} & =G_{2}-M B=d C_{1}-M B, \\
\widetilde{G}_{4} & =d C_{3}-C_{1} \wedge H+\frac{1}{2} M B \wedge B .
\end{aligned}
$$

\footnotetext{
${ }^{2}$ We use the notations of [ $\left[2 \overline{1}_{1}^{1}\right.$, section B.4] for differential forms.
} 
The scalar $M$ is an auxiliary field, and the above action reduces to ordinary (massless) Type IIA supergravity when $M=0$. The equation of motion for $C_{9}$ implies that $M$ must be (piecewise) constant. The equation from varying $M$, with $B=0$, is

$$
M={ }^{*} d C_{9},
$$

so this field is sometimes referred to as the RR scalar field strength. This suggests that 8-branes create discontinuities in the value of $M$. In fact, the configuration corresponding to an 8-brane located at $x^{9}=0$ is given by [i]

$$
\begin{aligned}
d s^{2} & =f^{-1 / 2}\left(x^{9}\right) d x^{\mu} d x^{\nu} \eta_{\mu \nu}+f^{1 / 2}\left(x^{9}\right)\left(d x^{9}\right)^{2} \\
e^{-4 \Phi\left(x^{9}\right)} & =f^{5}\left(x^{9}\right) \\
M\left(x^{9}\right) & =\mp f^{\prime}\left(x^{9}\right)
\end{aligned}
$$

where

$$
f\left(x^{9}\right)=c-m\left|x^{9}\right|
$$

Here $c$ and $m$ are positive constants. The 8-brane is a domain wall across which the value of $M$ jumps from $-m$ to $m$. The sign in the solution for $M$ is correlated with the chirality of the unbroken supersymmetry, and therefore differentiates an 8-brane from an anti-8-brane. In string theory the jump is quantized in units of the D8-brane charge $\mu_{8},{ }^{3}$ so for $2 k$ D 8 -branes we get

$$
M\left(x^{9}\right)=2 k \kappa_{10}^{2} \mu_{8} \epsilon\left(x^{9}\right)=\frac{k \epsilon\left(x^{9}\right)}{2 \pi \sqrt{\alpha^{\prime}}},
$$

where $\epsilon\left(x^{9}\right)$ is the unit step function.

Note that the above solution is ill-defined globally, since the dilaton, and therefore the string coupling $g_{s}=e^{\Phi}$, blows up at a finite distance from the 8-brane. ${ }^{4}$ However, as long as we stay close enough to the 8-brane the background is locally consistent. ${ }^{5}$ In particular this is the case in the decoupling limit we will consider.

Now consider a D2-brane along $\left(x^{1}, x^{2}\right)$, and at $x^{9}>0$, in the background of $2 k$ D8-branes. Supersymmetry is completely broken since there are six coordinates with mixed (ND) boundary conditions. The low-energy world-volume field theory of the D2-brane contains the usual $D=3 \mathcal{N}=8$ vector multiplet, i.e. a gauge field, seven adjoint scalars $X^{I}(I=3, \ldots, 9)$, and eight adjoint fermions, as well as $2 k$ massive fundamental fermions $\chi_{a}(a=1, \ldots, 2 k)$ from the D2-D8 strings. The action takes the form

$$
S_{D 2}=S_{S Y M}+S_{t o p}+S_{\chi}
$$

\footnotetext{
${ }^{3}$ The charge of a $\mathrm{D} p$-brane is given by $\mu_{p}=(2 \pi)^{-p}\left(\alpha^{\prime}\right)^{-(p+1) / 2}\left[2 \mathrm{i}_{1}^{\prime}\right]$.

${ }^{4}$ This was not the case for the solution originally written in [1], which corresponds to $m<0$ in our conventions. The latter presumably describes a combination of an orientifold 8-plane and seven D8-branes (plus images), which carries the same total charge as a single (anti) D8-brane.

${ }^{5}$ A completely consistent configuration of 8-branes requires the introduction of orientifold planes (as in Type IA string theory) to cut off the growth of the dilaton.
} 
where

$$
\begin{aligned}
S_{S Y M} & =-\frac{1}{g_{Y M_{3}}^{2}} \int d^{3} \sigma \sqrt{-g}\left[\frac{1}{4} g^{\alpha \alpha^{\prime}} g^{\beta \beta^{\prime}} F_{\alpha \beta} F_{\alpha^{\prime} \beta^{\prime}}+\frac{1}{2\left(2 \pi \alpha^{\prime}\right)^{2}} g^{\alpha \beta} g_{I J} \partial_{\alpha} X^{I} \partial_{\beta} X^{J}+\cdots\right], \\
S_{\text {top }} & =\mu_{2} \int\left[C_{3}+C_{1} \wedge\left(2 \pi \alpha^{\prime} F+B\right)\right],
\end{aligned}
$$

and

$$
S_{\chi}=\int d^{3} \sigma \bar{\chi}_{a}\left(i \not D+\frac{X^{9}}{2 \pi \alpha^{\prime}}\right) \chi_{a}
$$

and the YM coupling is given by $g_{Y M_{3}}^{2}=g_{s} / \sqrt{\alpha^{\prime}} \cdot{ }^{6}$

We now integrate out the fundamental fermions. This has two effects: it produces a Chern-Simons term for the gauge field via the parity anomaly $[2 \overline{2}, 1,2 \overline{2} \overline{1}$,

$$
S_{C S}=\frac{k}{4 \pi} \int d^{3} \sigma \epsilon^{\alpha \beta \gamma} A_{\alpha} \partial_{\beta} A_{\gamma}
$$

where $\epsilon^{012}=-\epsilon_{012}=-1$, and a potential for $X^{9}$. The precise form of the potential is not important for our purpose.

\subsection{Non-commutative D2-brane}

It is well-known that turning on a constant NSNS field, and taking an appropriate decoupling limit, deforms an ordinary D-brane world-volume gauge theory into a non-commutative gauge theory [i1 in. In our case we consider a constant NSNS field along $\left(x^{1}, x^{2}\right), B_{12}=-B_{21}=B$, which deforms the YMCS theory on the D2-brane to a non-commutative YMCS theory. In particular, the gauge part of the action becomes

$$
\begin{aligned}
S_{\text {gauge }}= & -\frac{1}{4 g_{Y M_{3}}^{\prime 2}} \int d^{3} \sigma \sqrt{-\hat{G}} \hat{G}^{\alpha \alpha^{\prime}} \hat{G}^{\beta \beta^{\prime}} \hat{F}_{\alpha \beta} * \hat{F}_{\alpha^{\prime} \beta^{\prime}}+ \\
& +\frac{k}{4 \pi} \int d^{3} \sigma \epsilon^{\alpha \beta \gamma}\left(\hat{A}_{\alpha} * \partial_{\beta} \hat{A}_{\gamma}+\frac{2 i}{3} \hat{A}_{\alpha} * \hat{A}_{\beta} * \hat{A}_{\gamma}\right)
\end{aligned}
$$

where $\hat{G}^{\alpha \beta}$ is the open string metric given in the Seiberg-Witten limit by ${ }^{7}$

$$
\hat{G}^{\alpha \beta}= \begin{cases}-\left(B^{-1} g B^{-1}\right)^{\alpha \beta} & \alpha, \beta=1,2 \\ g^{\alpha \beta} & \text { otherwise }\end{cases}
$$

and $g_{Y M_{3}}^{\prime}$ is the effective YM coupling defined by

$$
g_{Y M_{3}}^{\prime 2}=g_{Y M_{3}}^{2} \sqrt{\operatorname{det}\left(B g^{-1}\right)} .
$$

\footnotetext{
${ }^{6}$ For a D $p$-brane the YM coupling is $g_{Y M_{p+1}}^{2}=g_{s}(2 \pi)^{p-2}\left(\alpha^{\prime}\right)^{(p-3) / 2}$.

${ }^{7}$ Our convention for $B$ differs from [i] ${ }_{1}^{1} \overline{1}_{-1}^{\prime}$ in that $B=2 \pi \alpha^{\prime} B_{S W}$, so in the decoupling limit $B \sim \alpha^{\prime}$.
} 
The star product is defined in terms of the non-commutativity parameter ${ }^{8}$

$$
\theta^{\alpha \beta}=2 \pi \alpha^{\prime}\left(B^{-1}\right)^{\alpha \beta}
$$

This is most easily understood by turning on the $B$ field before integrating out the fundamental fermions. The resulting non-commutative YM theory with fundamental (Dirac) fermions exhibits a parity anomaly analogous to the commutative case, and a one-loop non-commutative Chern-Simons term is produced [2 $2 \overline{4} \overline{4}$.

In the Seiberg-Witten zero-slope limit the theory contains both a non-commutative YM term and a non-commutative CS term, as well as scalar fields and fermions. We shall instead consider a slight modification of this limit, in which

$$
\begin{aligned}
g_{\alpha \beta} & \sim\left(\alpha^{\prime}\right)^{2+\delta} \quad(\alpha, \beta=1,2) \\
g_{s} & \sim\left(\alpha^{\prime}\right)^{3 / 2+\epsilon},
\end{aligned}
$$

where $\delta>\epsilon>0$. The Seiberg-Witten limit corresponds to the case that $\delta=\epsilon=0$, which keeps $\theta^{\alpha \beta}, \hat{G}^{\alpha \beta}$ and $g_{Y M_{3}}^{\prime}$ finite. In the new limit $\theta^{\alpha \beta}$ remains finite, but $\hat{G}^{\alpha \beta}$ and $g_{Y M_{3}}^{\prime}$ scale as

$$
g_{Y M_{3}}^{\prime 2} \sim\left(\alpha^{\prime}\right)^{\epsilon-\delta}, \quad \hat{G}^{\alpha \beta} \sim\left(\alpha^{\prime}\right)^{\delta} \quad(\alpha, \beta=1,2)
$$

In this limit the YM term vanishes, since

$$
\frac{1}{2 g_{Y M_{3}}^{\prime 2}} \sqrt{-\hat{G}} \hat{G}^{11} \hat{G}^{22} \hat{F}_{12} * \hat{F}_{12} \sim\left(\alpha^{\prime}\right)^{2 \delta-\epsilon}
$$

and

$$
\frac{1}{2 g_{Y M_{3}}^{\prime 2}} \sqrt{-\hat{G}} \hat{G}^{00} \hat{G}^{\alpha \alpha} \partial_{t} \hat{A}_{\alpha} * \partial_{t} \hat{A}_{\alpha} \sim\left(\alpha^{\prime}\right)^{\delta-\epsilon} .
$$

In addition the scalar fields decouple, since the effective coupling constant for the canonically normalized fields

$$
\phi^{I}=\frac{(-\hat{G})^{1 / 4}}{g_{Y M_{3}}^{\prime}} \frac{\sqrt{g_{I I}}}{2 \pi \alpha^{\prime}} X^{I}
$$

is given by $g_{Y M_{3}}^{\prime} /(-\hat{G})^{1 / 4} \sim\left(\alpha^{\prime}\right)^{\epsilon / 2}$. Thus any interaction term written in terms of $\hat{A}_{\alpha}$ and $\phi^{I}$ vanishes, because this effective coupling constant vanishes, and $\hat{G}^{\alpha \beta}$, which

${ }^{8}$ Our convention for the star product is

$$
f(x) * g(x)=\left.\exp \left(-\frac{i}{2} \theta^{\alpha \beta} \frac{\partial}{\partial \xi^{\alpha}} \frac{\partial}{\partial \zeta^{\beta}}\right) f(x+\xi) g(x+\zeta)\right|_{\xi=\zeta=0} .
$$

The ordering of the open string space-time fields corresponding to the vertex operators $A(X(\tau))$ and $B\left(X\left(\tau^{\prime}\right)\right)$ is taken to be $A(X) B(X)$ when $\tau<\tau^{\prime}$. This is the convention taken in [400, $33 \overline{1}$, and is different from the one in [1 $\left.1 i_{1}^{1}\right]$. 
contracts indices from the non-commutative gauge field, vanishes. It is straightforward to extend the argument to include the fermions. This leaves us with the pure non-commutative Chern-Simons (NCCS) theory,

$$
S_{N C C S}=\frac{k}{4 \pi} \int d^{3} \sigma \epsilon^{\alpha \beta \gamma}\left(\hat{A}_{\alpha} * \partial_{\beta} \hat{A}_{\gamma}+\frac{2 i}{3} \hat{A}_{\alpha} * \hat{A}_{\beta} * \hat{A}_{\gamma}\right)
$$

Note also that the limit in $(\overline{2} .15 i)$ also implies that in the supergravity solu-

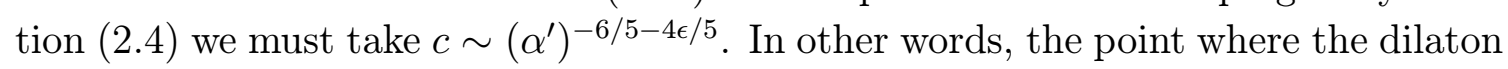
diverges is taken to infinity, and $g_{s}$ goes to zero at the location of the D2-brane. The supergravity soultion is therefore well-defined.

\subsection{Quantum Hall fluid}

There is an apparent problem with the previous discussion. Unlike massless supergravity, the equations of motion of massive supergravity ( $(2 . \overline{1} \cdot \overline{1})$ do not admit a solution with a nontrivial constant $B$ field and all other fields trivial. Another way to see this is that the $B$ field induces sources for the RR fields inside the world-volume of the 8-branes. In the simplest case of a single non-trivial constant component $B_{12}=B$, and a trivial $C_{3}$, the equation of motion for the NSNS field is given by

$$
d\left(e^{-2 \Phi *} H\right)=M^{*}\left(G_{2}-M B\right) .
$$

Since $H$ vanishes we find that the solution requires a $G_{2}$ flux along $\left(x^{1}, x^{2}\right)$

$$
G_{2}=M B=\frac{k B}{2 \pi \sqrt{\alpha^{\prime}}} \epsilon\left(x^{9}\right) .
$$

This can also be understood from the fact that the constant $B$ field induces a uniform D6-brane charge density per unit area in the $\left(x^{1}, x^{2}\right)$ plane in the world-volume of the D8-brane. From the coupling

$$
\mu_{8} \int C_{7} \wedge B
$$

in the D8-brane world-volume theory, we deduce that the D6-brane charge density for $2 k$ D8-branes is

$$
\rho_{6}=2 k \mu_{8} B=\frac{2 k B}{(2 \pi)^{8}\left(\alpha^{\prime}\right)^{9 / 2}} .
$$

The resulting magnetic field is given by

$$
G_{2}=\frac{1}{2}\left(2 \kappa_{10}^{2}\right) \rho_{6} \epsilon\left(x^{9}\right)=\frac{k B}{2 \pi \sqrt{\alpha^{\prime}}} \epsilon\left(x^{9}\right)
$$

in agreement with $(\underline{2} . \overline{2} \overline{2}$ in). We will assume that this relation continues to hold in the presence of the D2-brane. 


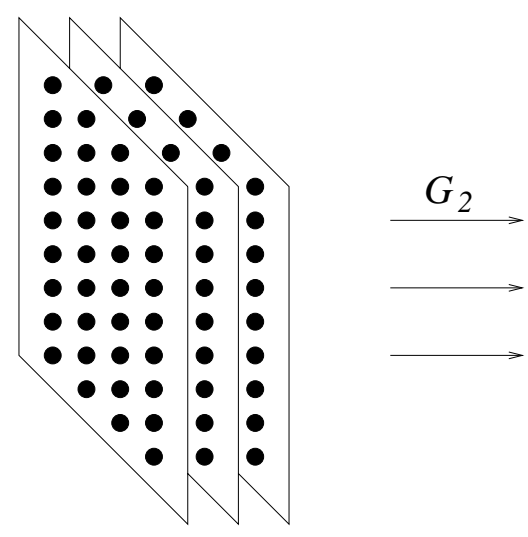

$2 k$ D8-branes

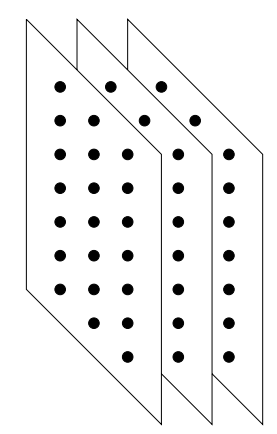

$n$ D2-branes

Figure 1: Brane configuration of the quantum Hall fluid with $\nu=n / k$. A uniform $B$ field induces D-particles (small circles) inside the D2-branes, and D6-branes (large circles) inside the D8-branes. The D6-branes give rise to a uniform magnetic RR field $G_{2}$.

This is where the problem becomes a virtue. The $B$ field induces a uniform D-particle charge density per unit area in the membrane given by

$$
\rho_{0}=\mu_{2} B=\frac{B}{(2 \pi)^{2}\left(\alpha^{\prime}\right)^{3 / 2}} .
$$

The non-commutative membrane can therefore be thought of as a two-dimensional fluid of RR-charged D-particles in a background RR magnetic field, i.e. a quantum Hall fluid. The filling fraction $\nu$ is defined as the ratio of the carrier density to the degeneracy of the Landau level. The latter is given by $\mu_{0} G_{2} /(2 \pi)$, so the filling fraction is

$$
\nu=\frac{\rho_{0} / \mu_{0}}{\mu_{0}\left|G_{2}\right| /(2 \pi)}=\frac{1}{k} .
$$

This establishes Susskind's conjecture that a quantum Hall fluid at filling fraction $1 / k$ is described by a NCCS theory at level $k[4]$. In this approach it is clear why the inverse filling fraction is quantized; it simply corresponds to the quantized value of the RR scalar field strength $M$, or equivalently to (half) the number of D8-branes. It is less clear at this stage whether the D-particles in the membrane behave like fermions or bosons, and how this property correlates with $k$. This will be clarified in the matrix model picture in the next section.

\subsection{Multiple D2-branes}

It was also conjectured that the theory for a quantum Hall fluid with a filling fraction $\nu=n / k$ would be a level $k U(n)$ NCCS theory $\left[\begin{array}{l}4 \\ i\end{array}\right]$. In our construction this is a trivial generalization of $\nu=1 / k$ case. Consider $n$ D2-branes in massive supergravity with $2 k$ D8-branes. The resulting world-volume theory is $U(n)$ YMCS theory with adjoint matter, and a CS coefficient $k$. In the presence of the $B$ field, and in our scaling 
limit, this becomes a level $k U(n)$ NCCS theory. The RR magnetic field is still given by $(2 \overline{2} . \overline{2} \overline{2})$, but the D-particle charge density is now

$$
\rho_{0}=n \mu_{2} B
$$

so the filling fraction is

$$
\nu=\frac{n}{k} .
$$

The challenge is to understand, in this picture, which fractions actually exhibit a mass-gap, and why there is a hierarchy of fractions.

\section{D-particles and the Chern-Simons matrix model}

It is well-known that non-commutative Yang-Mills theory on a $\mathrm{D} p$-brane with $2 n$ non-commutative directions can be obtained from a non-commutative configuration of $\mathrm{D}(p-2 n)$-branes [i] massless Type IIA D2-brane is obtained by considering fluctuations about a noncommutative configuration in the D-particle matrix model. Similarly, NCCS theory can also be obtained from a one-dimensional matrix model [i] $\overline{1} \overline{8}]$

$$
S=\int d t \operatorname{Tr}\left[-k A+\frac{k}{2}\left(\theta^{-1}\right)_{i j} X^{i} D_{0} X^{j}\right]
$$

expanded about a non-commutative background

$$
X^{i}=x^{i}+\theta^{i j} \hat{A}_{j}
$$

where

$$
\left[x^{i}, x^{j}\right]=-i \theta^{i j} .
$$

To make this consistent for finite $N$, it was proposed in [i]

$$
i \int d t \psi^{\dagger} D_{0} \psi
$$

where $\psi$ is a complex boson transforming in the fundamental representation of $\mathrm{U}(N)$. We will show that this matrix model can be realized by D-particles in massive IIA supergravity with a $B$-field in the scaling limit described in the previous section.

\subsection{D-particles in massive IIA supergravity}

It has been known for quite some time that there are subtleties involved in introducing D-particles in massive IIA supergravity, i.e. in the presence of D8-branes $\left[2 \overline{2} \overline{5}_{-}^{\prime}, 1 \overline{2} \overline{6}\right.$ - In particular, charge conservation requires strings to end on D-particles, whereas this 
is forbidden in massless IIA [27]. In the spacetime picture this follows from the equation of motion for $B$, which for trivial background fields becomes

$$
d\left(e^{-2 \Phi *} H\right)=M^{*} G_{2}+\frac{2 \kappa_{10}^{2}}{2 \pi \alpha^{\prime}} n_{s} \delta_{8},
$$

where the second term corresponds to $n_{s}$ fundamental string sources. Integrating over an 8-sphere surrounding $N$ D-particles we find that

$$
n_{s}=-2 \pi \alpha^{\prime} M N \mu_{0}=-k N \epsilon\left(x^{9}\right),
$$

where we have used the value of $M$ for $2 k$ D8-branes at $x^{9}=0\left(\underline{2} . \overline{6}_{i}^{\prime}\right)$. It follows that $k$ strings are attached to each D-particle, and their orientation depends on which side of the D8-branes the D-particle is. Alternatively, if a string ends on any other Dbrane, the above equation implies that its end carries $1 / k$ units of $\mathrm{D}$-particle charge. This fact will be important in the next section.

Another way to see that strings are attached to the D-particle is via the worldline theory [2] $\overline{2} \overline{8}]$. In this picture the end of the string is a source for the world-line electric field, and is necessary in order to cancel an induced electric charge due to the background D8-branes. In the usual decoupling limit

$$
\alpha^{\prime} \rightarrow 0, g_{i j} \sim\left(\alpha^{\prime}\right)^{2}, g_{s} \sim\left(\alpha^{\prime}\right)^{3 / 2}, \text { everything else } \sim O(1),
$$

the world-line theory for $N$ D-particles is given by [i2 $2 \overline{6}]$

$$
\begin{aligned}
S= & \frac{1}{g_{Y M_{1}}^{2}} \int d t \operatorname{Tr}\left[\frac{1}{2\left(2 \pi \alpha^{\prime}\right)^{2}} g_{i j} D_{0} X^{i} D_{0} X^{j}+\frac{1}{4\left(2 \pi \alpha^{\prime}\right)^{4}} g_{i k} g_{j l}\left[X^{i}, X^{j}\right]\left[X^{k}, X^{l}\right]+\cdots\right]+ \\
& +\int d t\left\{-\operatorname{Tr}\left[k A_{0}+\frac{k X^{9}}{2 \pi \alpha^{\prime}}\right]+\psi_{a}^{\dagger}\left(i D_{0}-\frac{X^{9}}{2 \pi \alpha^{\prime}}\right) \psi_{a}\right\}
\end{aligned}
$$

where $\psi_{a}(a=1, \ldots, 2 k)$ are $N$-component vectors of fermions coming from the (R sector of the) 0-8 strings, and we have suppressed the terms involving the fermionic partners of $X^{i}$. Each of the fundamental fermions contributes a CS term with coefficient $-1 / 2$, as well as a linear $X^{9}$ potential, to the one-loop effective action $\left[2 \overline{2} \overline{9_{1}}, \overline{2} \overline{2} \overline{\hat{Q}}\right]$. The former corresponds to an induced charge, and the latter to a non-vanishing repulsive force. In order to cancel these we must add a bare CS term and an attractive potential as above. The former is interpreted as the charge at the end of the string, and the latter is due to the tension of the string.

\subsection{The Chern-Simons matrix model}

Now consider turning on a constant $B$ field along $\left(x^{1}, x^{2}\right)$. As we have seen in the previous section, this requires turning on a constant $G_{2}$ along $\left(x^{1}, x^{2}\right)$ as well. This introduces additional terms in the equation of motion for $B$,

$$
\begin{aligned}
d\left(e^{-2 \Phi *} H\right)= & M^{*} G_{2}-M^{2 *} B-M^{*} d C_{3} \wedge B+ \\
& +d\left({ }^{*} d C_{3} \wedge C_{1}-\frac{1}{2} C_{3} \wedge d C_{3}\right)+\frac{2 \kappa_{10}^{2}}{2 \pi \alpha^{\prime}} n_{s} \delta_{8} .
\end{aligned}
$$


In particular, the third term contributes to the integral over an eight-sphere at infinity if D2-branes are present, and one gets

$$
n_{s}=-k N+\frac{k B}{(2 \pi)^{2} \alpha^{\prime}} n A=-k N+\frac{G_{2}}{2 \pi \sqrt{\alpha^{\prime}}} n A
$$

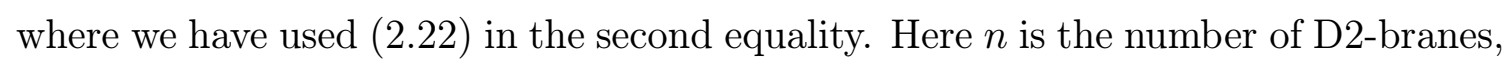
and $A$ is their (infinite) area. Using the definition of the filling fraction $\nu\left(2.2 \overline{2} \bar{z}_{1}\right)$, we can rewrite this condition as

$$
n_{s}=-N\left(k-\frac{n}{\nu}\right)
$$

If $n=0$ the situation is the same as before, and each D-particle has $k$ strings attached. For $n \neq 0$ the number of strings depends on the filling fraction. In particular for $\nu=n / k$ we find $n_{s}=0$. Thus no strings are attached to the Dparticles in the quantum Hall fluid phase. In the next section we will consider a more general situation in which the filling fraction is different from this value, which requires strings to end on the D2-branes.

The world-line theory is also modified in the presence of $B$ and $G_{2}$. First, there will be an additional $X^{9}$ potential due to the induced D6-branes. More importantly

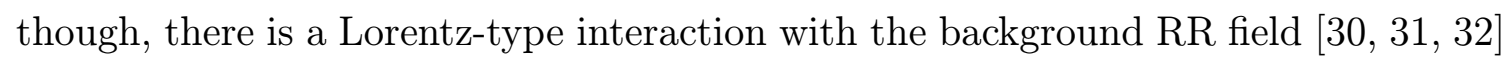

$$
S_{G_{2}}=\frac{\mu_{0}}{2} \int d t\left(G_{2}\right)_{i j} \operatorname{Tr}\left[X^{i} D_{0} X^{j}\right]
$$

Now consider the decoupling limit (2.15 $\left.\overline{1}_{1}^{\prime}\right)$, in which $g_{Y M_{1}}^{2}=g_{s}(2 \pi)^{-2}\left(\alpha^{\prime}\right)^{-3 / 2} \sim\left(\alpha^{\prime}\right)^{\epsilon}$. In this limit the fields $X^{I}$, with $I=3, \ldots, 9$, and the adjoint fermions decouple completely. In addition, the CS term (3.3.1 and $X^{2}$, and the only non-trivial part of the matrix model is given by

$$
\begin{aligned}
S & =\int d t\left\{\operatorname{Tr}\left[\frac{1}{2 \sqrt{\alpha^{\prime}}}\left(G_{2}\right)_{i j} X^{i} D_{0} X^{j}-k A_{0}\right]+i \psi_{a}^{\dagger} D_{0} \psi_{a}\right\} \\
& =\int d t\left\{\operatorname{Tr}\left[\frac{k}{2}\left(\theta^{-1}\right)_{i j} X^{i} D_{0} X^{j}-k A_{0}\right]+i \psi_{a}^{\dagger} D_{0} \psi_{a}\right\},
\end{aligned}
$$

where we have used ( $\left(\overline{2} . \overline{2} \overline{2}_{1}^{\prime}\right)$ and $\left(\overline{2} . \overline{1} \overline{4}^{\prime}\right)$ in the second equality. This is almost identical to the finite $N$ CS matrix model discussed in [1] 91 . The only difference is that here the fields $\psi_{a}$ are fermions, whereas in [1] 9 ] they are treated as bosons. The former is more natural, given the form of their action. Recall that these fields correspond to 0-8 strings.

\subsection{Solutions}

The dynamics of the matrix model in ( $\overline{3} . \overline{1} \cdot \overline{3})$ is determined completely from the Gauss law constraint,

$$
-i\left(\alpha^{\prime}\right)^{-1 / 2} G_{2}\left[X^{1}, X^{2}\right]_{m n}+\psi_{a, m} \psi_{a, n}^{\dagger}=k \delta_{m n}
$$


Note that for $G_{2}=0$ we get

$$
\psi_{a, m} \psi_{a, n}^{\dagger}=k \delta_{m n} .
$$

This is just the statement that $k$ strings are attached to each D-particle.

For finite $N$ the trace of the constraint is always

$$
\operatorname{Tr} \psi_{a} \psi_{a}^{\dagger}=N k
$$

since the trace of the commutator vanishes. However, when $N \rightarrow \infty$ more general solutions to the trace constraint are possible. In particular, the quantum Hall fluid corresponds to a non-commutative D2-brane, which is described by a D-particle configuration $X^{1}=x^{1}, X^{2}=x^{2}$, and $X^{I}=0(I=3, \ldots, 9)$, in which

$$
\left[x^{1}, x^{2}\right]_{m n}=-i \theta^{12} \delta_{m n}=i \theta \delta_{m n},
$$

where we have defined $\theta=-\theta^{12}$. It then follows from $\left(2 \underline{2} . \overline{1} \overline{4}_{1}\right)$ and $(\underline{2} . \overline{2} \overline{2})$ that

$$
\psi_{a} \psi_{a}^{\dagger}=0
$$

namely that there are no strings. This is consistent with what was found in the spacetime viewpoint $\left(\overline{\underline{3}} . \overline{1} \overline{1} \overline{1}^{\prime}\right)$.

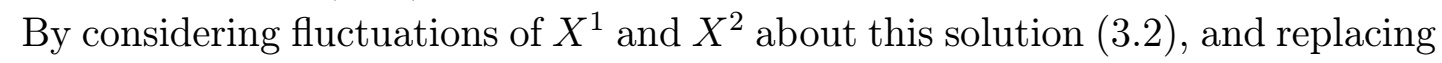

$$
\operatorname{Tr} \rightarrow \frac{\operatorname{Pf}\left(\theta^{-1}\right)}{2 \pi} \int d x^{1} d x^{2}=\frac{1}{2 \pi \theta} \int d x^{1} d x^{2},
$$

one obtains NCCS theory [4]. The D-particle density per unit area in this configuration is $1 /(2 \pi \theta)$, so the filling fraction is $1 / k$, in agreement with the result in section $i_{-i}^{2}$.

The advantage of the matrix model approach is that it allows us to address the question of the statistics of the D-particles, i.e. the electrons of the QH fluid. In general, D-particles have rather complicated "non-abelian" statistics, due to the fact that their positions are described by matrices rather than numbers. However, in the non-commutative configuration described above the effective two-dimensional statistics in the $\left(x^{1}, x^{2}\right)$ plane simplifies. As shown in [4], the phase associated with exchanging two D-particles in the D2-brane configuration is given by $\exp (i \pi k)$. This was used in to argue that $k$ should be quantized. In our case $k$ is (half) the number of D8-branes, so it is manifestly quantized. We therefore see that the Dparticles behave as bosons if $k$ is even, and fermions if $k$ is odd.

\section{Quasiparticles and quasiholes}

The quantum Hall fluid at filling fraction $1 / k$ (with $k$ odd) is a stable state with a mass-gap [i] 
subtracting a unit of magnetic flux, and thereby slightly shifting the filling fraction. The former is known as a quasihole, and the latter as a quasiparticle. Due to the incompressibility of the quantum Hall fluid, it can be shown that these excitations effectively carry a fractional charge $\mp 1 / k$, and therefore exhibit fractional statistics. The total charge of the fluid remains the same, but the additional flux causes a redistribution of the charge density. In this section we will identify the quasiparticles and quasiholes in our string picture, and show that they have the correct charge and statistics.

\subsection{Space-time picture}

Let us begin with a single D2-brane, and a filling fraction $\nu=1 / k$. Now consider a shift in the RR magnetic field corresponding to one flux quantum, i.e.

$$
G_{2} \rightarrow G_{2}+\Delta G_{2}
$$

where $\Delta G_{2}= \pm 2 \pi \sqrt{\alpha^{\prime}} / A$. This can be achieved by creating a D6- $\overline{\mathrm{D} 6}$ pair, and moving the D6 (in the + case) or the $\overline{\mathrm{D} 6}$ (in the - case) across the D2-brane (figure 2). Since the total D-particle number $N$ is kept fixed, this changes the filling fraction to

$$
\nu^{\prime}=\frac{1}{k \pm \frac{1}{N}} .
$$

The condition ( $\left(\bar{b}_{3} \overline{1} \overline{1}_{1}^{\prime}\right)$ now requires $n_{s}= \pm 1$. This can also be understood from the fact that when the D6 (or $\overline{\mathrm{D} 6}$ ) crosses the D2-brane a string is created between them. We therefore identify the quasiparticle (quasihole) with a string ending on the D2brane. This also explains, from the spacetime point of view, why quasiparticles and quasiholes correspond to sources in the world-volume NCCS theory [A].

The above result suggests that the quasiparticle and quasihole carry a fractional D-particle number $\pm 1 / k$, since a whole D-particle (outside the D2-brane) requires $k$ strings. Indeed, if $G_{2}=B=0$, the equation of motion for $B\left({ }_{3} . \bar{y}_{1}\right)$ implies that the end of a string on any D-brane in massive supergravity carries $1 / k$ units of D-particle charge. In our case the background fields are non-trivial, and the total D-particle charge is held fixed. The latter can be thought of as coming from two components

$$
N=(N+\Delta N)-\Delta N
$$

where $\Delta N= \pm 1 / k$. The first component corresponds to a quantum Hall fluid with filling fraction given by

$$
\nu=\frac{2 \pi \sqrt{\alpha^{\prime}}}{A} \frac{N+\Delta N}{G_{2}+\Delta G_{2}}=\frac{1}{k},
$$

and the second component is the quasihole (or quasiparticle). The idea is that when we shift $G_{2}$ the D-particle density changes to maintain the quantized filling fraction. Conservation of total D-particle charge then requires a charge deficit (or excess) 


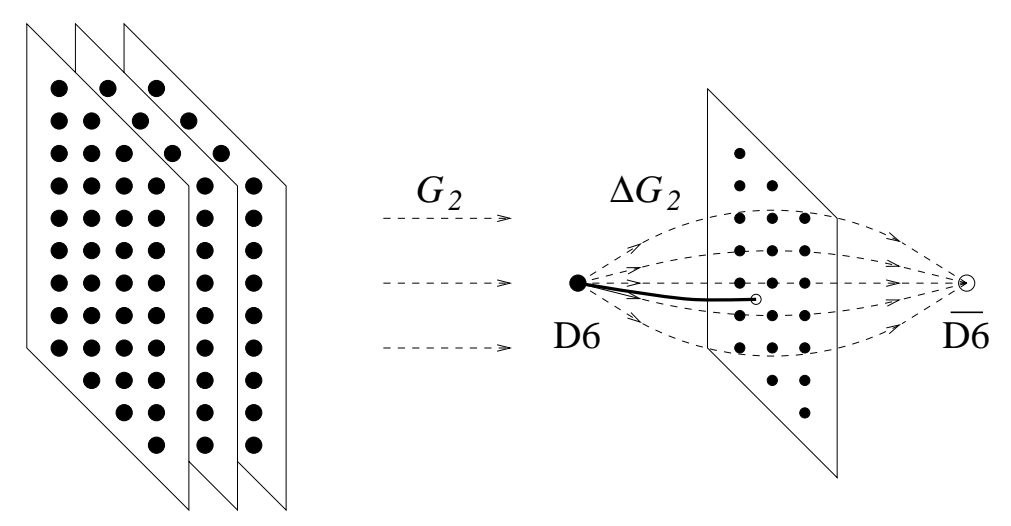

Figure 2: Brane configuration of a quasihole. The D6- $\overline{\mathrm{D} 6}$ pair adds one unit of $G_{2}$ flux, as well as a D2-D6 string. The end of the string on the D2-brane carries a deficit RR charge $\mu_{0} / k$, represented by the unfilled circle.

somewhere, and this is provided by the end of the string. The new state can be thought of as consisting of two components

$$
\Psi=\Psi_{f} \cdot \Psi_{q p},
$$

where $\Psi_{f}$ describes the fluid at $\nu=1 / k$, and $\Psi_{q p}$ describes the quasiparticle. This will be made somewhat more quantitative in the matrix model approach.

Consider now a pair of quasiparticles corresponding to two strings ending on the D2-brane at different positions. When we move one of the string ends around the other, the quasiparticle wavefunction acquires an Aharonov-Bohm phase. Relative to the case with a single quasiparticle, the phase given by

$$
\phi=A \Delta G_{2} \cdot \frac{\mu_{0}}{k}= \pm \frac{2 \pi}{k} .
$$

Thus the quasiparticles exhibit fractional statistics.

\subsection{Matrix model picture}

The ground state of a quantum Hall fluid at filling fraction $1 / k$ corresponds to a solution of $(\overline{\underline{3}} \cdot \overline{1} \overline{4})$ with

$$
\begin{aligned}
\psi_{a} \psi_{a}^{\dagger} & =0 \\
{\left[X^{i}, X^{j}\right] } & =-i \theta^{i j} \mathbf{1},
\end{aligned}
$$

where

$$
\theta^{i j}=2 \pi \alpha^{\prime}\left(B^{-1}\right)^{i j}=\sqrt{\alpha^{\prime}} k\left(G_{2}^{-1}\right)^{i j} .
$$

This corresponds to a non-commutative D2-brane with a uniform D-particle density given by

$$
\frac{N}{A}=\frac{\left(\theta^{-1}\right)_{12}}{2 \pi}
$$

and no strings. 
A quasiparticle (or quasihole) corresponds to adding an additional unit of $G_{2}$ flux, while maintaining the same filling fraction for the fluid component, and keeping the total D-particle charge fixed. The latter condition requires a string to end on the D2-brane, which in the matrix model corresponds to $\operatorname{Tr} \psi_{a} \psi_{a}^{\dagger}=\psi_{a, n} \psi_{a, n}^{\dagger}=\mp 1$. For example, if we choose

$$
\psi_{a} \psi_{a}^{\dagger}=\mp|0\rangle\langle 0|
$$

Gauss' law is solved by

$$
\left[X^{i}, X^{j}\right]=-i\left(\theta^{\prime}\right)^{i j}\left(\mathbf{1} \pm \frac{1}{k}|0\rangle\langle 0|\right)
$$

where

$$
\left(\theta^{\prime}\right)^{i j}=\sqrt{\alpha^{\prime}} k\left(\frac{1}{G_{2}+\Delta G_{2}}\right)^{i j} .
$$

This agrees with Susskind's prescription of introducing by hand a source term to Gauss' law in order to describe a quasiparticle [i. In our case the "source" is provided by the field $\psi$, which fits with our identification of the quasiparticle and

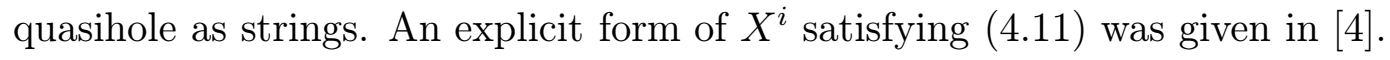

\subsection{Charge distribution}

Given any D-particle configuration $X^{i}$, one could in principle compute the distribu-

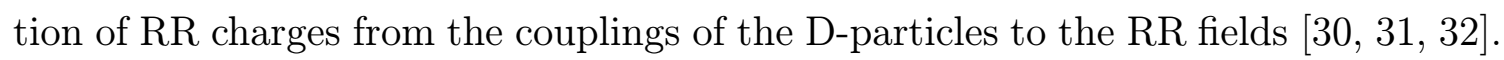
In particular, the (Fourier transform of) the D-particle density is given by

$$
J_{D 0}(p)=\operatorname{Tr} e^{i p X} .
$$

This is derived from the coupling to $\left(C_{1}\right)_{0}$ :

$$
\mu_{0} \int d t \operatorname{Tr}\left(C_{1}\right)_{0}(X)=\mu_{0} \int d t \int \frac{d^{2} p}{(2 \pi)^{2}}\left(\tilde{C}_{1}\right)_{0}(-p) J_{D 0}(p)
$$

where

$$
\left(\tilde{C}_{1}\right)_{0}(p)=\int d^{2} x e^{i p x}\left(C_{1}\right)_{0}(x) .
$$

The total D-particle number is given by $J_{D 0}(0)=\operatorname{Tr} \mathbf{1}=N$. If we map the matrix configuration of $X^{i}$ to the non-commutative gauge field configuration $\hat{A}_{i}$, the $\mathrm{RR}$ currents give the coupling of the non-commutative gauge field to the RR poten-

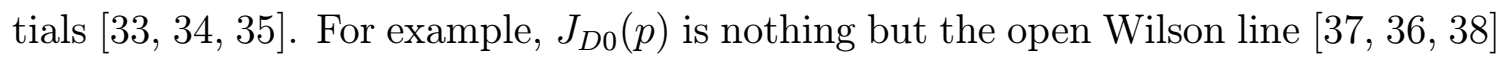

$$
J_{D 0}(p)=\frac{\operatorname{Pf}\left(\theta^{-1}\right)}{2 \pi} \int d^{2} x\left[e^{i p x} P \exp \left(i \int_{0}^{1} \hat{A}_{i}(x+l \tau) l^{i} d \tau\right)\right]
$$

where $l^{i}=p_{j} \theta^{j i}$ and the star product is implicit. We can use the RR currents to study the D-brane charge distribution of non-commutative solitons as was done 
in $\left[39.9\right.$. We would like to compute the $p$ dependence of $J_{D 0}$, and thereby the charge distribution, for the case of the quasiparticle (or quasihole) ( $\left.\overline{4} . \overline{1} \overline{1} \overline{1}^{\prime}\right)$.

It turns out to be technically challenging to compute $J_{D 0}(p)$ using the explicit

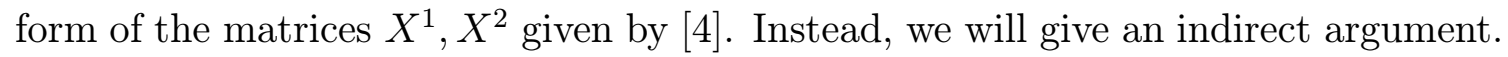
It was shown in $[3 \overline{3}, 13 \overline{4}, 150$, topological, in the sense that its value does not change for a small variation of the configuration $\delta X^{i}$. This is physically reasonable because the D2-brane charge does not change for a small fluctuation of the non-commutative gauge field. The explicit form of $J^{0 i j}(p)$ is derived from the coupling

$$
-\frac{i}{2} \frac{\mu_{0}}{2 \pi \alpha^{\prime}} \int d t \operatorname{Tr} C_{0 i j}(X)\left[X^{i}, X^{j}\right]=\mu_{2} \int d t \int \frac{d^{2} p}{(2 \pi)^{2}} \tilde{C}_{0 i j}(-p) J^{0 i j}(p),
$$

and is given by

$$
J^{0 i j}(p)=-i \pi \operatorname{Tr}\left[X^{i}, X^{j}\right] e^{i p X} .
$$

For a configuration with a single D2-brane

$$
J^{0 i j}(p)=-i \pi \operatorname{Tr}\left[X^{i}, X^{j}\right] e^{i p X}=\frac{1}{2}(2 \pi)^{2} \epsilon^{i j} \delta(p) .
$$

For the quasiparticle solution ( $\left({ }_{-}^{A} . \overline{1} \overline{1}_{1}^{\prime}\right)$ this gives

$$
\operatorname{Tr}\left(\theta^{\prime}\right)^{i j}\left(\mathbf{1} \pm \frac{1}{k}|0\rangle\langle 0|\right) e^{i p X}=-2 \pi \epsilon^{i j} \delta(p) .
$$

The D-particle density is therefore given by

$$
J_{D 0}(p)=\operatorname{Tr} e^{i p X}=2 \pi\left(\theta^{\prime-1}\right)_{12} \delta(p) \mp \frac{1}{k}\left\langle 0\left|e^{i p X}\right| 0\right\rangle .
$$

The first term corresponds to a uniform distribution with density $1 /\left(2 \pi \theta^{\prime}\right)$, and the second term corresponds to the $1 / k$ excess (or deficit) charge carried by the quasiparticle (quasihole), which compensates for the change in the uniform distribution from $1 /(2 \pi \theta)$. We can easily compute this term in two extreme limits. For $k \rightarrow \infty$, the configuration reduces to $\left({ }_{-}^{\prime} \bar{A}_{-} \overline{7}_{1}^{\prime}\right)$, and the matrices can be expressed in terms of creation and annihilation operators

$$
X^{1}=\sqrt{\frac{\theta}{2}}\left(a+a^{\dagger}\right), \quad X^{2}=-i \sqrt{\frac{\theta}{2}}\left(a-a^{\dagger}\right) .
$$

We therefore find that

$$
\left\langle 0\left|e^{i p X}\right| 0\right\rangle=\exp \left[-\frac{\theta}{4}\left(p_{1}^{2}+p_{2}^{2}\right)\right],
$$

\footnotetext{
${ }^{9}$ In the Seiberg-Witten limit, there is no ambiguity in the D-brane charge distribution coming from field redefinition of RR potentials when we set at least one transverse scalar to be zero. The closed string on-shell condition can then be satisfied for an arbitrary momentum in the noncommutative directions $[\overline{4} \overline{0}]$. We thank $\mathrm{H}$. Ooguri for the discussion on this point.
} 
and the coordinate space charge distribution is gaussian. For $k=1$, on the other hand, the computation reduces to the one done in [i39], and the momentum space charge distribution is given by

$$
\left\langle 0\left|e^{i p X}\right| 0\right\rangle=1
$$

so in coordinate space it is $\delta(x)$. Since the charge deficit (or excess) is localized in both limits, we expect that it is localized in general.

The solution corresponding to a quasiparticle in ( $\left(\bar{A} . \overline{1} \bar{O}_{1}^{\prime}\right)$ and $\left(\bar{A} . \overline{1} \overline{1}_{1}^{\prime}\right)$ is not unique. For example, one can choose $\psi_{a} \psi_{a}^{\dagger}=|1\rangle\langle 1|$, and similarly replace ( $\left.\bar{A}_{-}^{\prime} \overline{1} \overline{1}_{1}^{\prime}\right)$. The precise form of the charge distribution of the quasiparticle changes, but the total charge deficit (or excess) is still $1 / k$.

Multi-quasiparticle (quasihole) states can be described by configurations of the form

$$
\begin{aligned}
\psi_{a} \psi_{a}^{\dagger} & =\sum_{m=0}^{N} c_{m}|m\rangle\langle m| \\
{\left[X^{i}, X^{j}\right] } & =-i\left(\theta^{\prime}\right)^{i j}\left(\mathbf{1}-\sum_{m=0}^{N} c_{m}|m\rangle\langle m|\right) .
\end{aligned}
$$

The total charge deficit (or excess) is simply given by $\operatorname{Tr} \psi_{a} \psi_{a}^{\dagger} / k=\sum c_{m} / k$. However the entire charge is concentrated at the origin. It would be interesting to find multi-centered solutions. In particular, one would like to identify the collective coordinates of multi-quasiparticle states, just as in the case of non-commutative solitons in [ATI]. This would be particularly useful in verifying the statistical properties of the quasiparticles in the matrix model, as was done for the D-particles in [i]

\section{Conclusions and outlook}

In this paper we have derived the correspondence between NCCS theory and the quantum Hall fluid. The brane picture which we have presented, namely a D2-brane in massive IIA string theory with a constant $B$ field background, in an appropriate decoupling limit, can be viewed in two ways. In the spacetime viewpoint the system is seen as a two-dimensional fluid of charged particles in a uniform background magnetic field, where the charged particles are D-particles, the magnetic field is the RR field $G_{2}$, and the fluid corresponds to a non-commutative configuration of D-particles forming a D2-brane. At the same time, the D2-brane world-volume theory is a pure NCCS gauge theory.

The brane picture naturally explains various aspects of the quantum Hall fluid, such as the quantization of the filling fraction, and the charge and statistics of the quasiparticle and quasihole excitations. Note, however, that since the hamiltonian 
of the NCCS theory (as well as the matrix model) vanishes, all the allowed states are degenerate in energy. In particular, the quasiparticle and quasihole excitations are massless in this context. It therefore remains unclear how to obtain a non-vanishing mass gap. One possibility is that additional interactions must be added to the action. In fact, the string construction gives a definite prescription for this; if we set $\epsilon=\delta=0$ in $\left(2.155_{1}^{\prime}\right)$, we recover the ordinary Seiberg-Witten limit, in which the theory has a finite YM term, as well as coupled scalars and fermions. It would be interesting to study this situation.

It would also be interesting if the string realization gives some insights into the transition to a Wigner crystal phase at low filling fraction. The relevant physical gauge-invariant observables for this problem are correlation functions of the Dparticle density $J_{D 0}(p)$. It was suggested in [青] to calculate the correlation functions by mapping to the noncommutative gauge theory in a power series expansion in $\theta$.

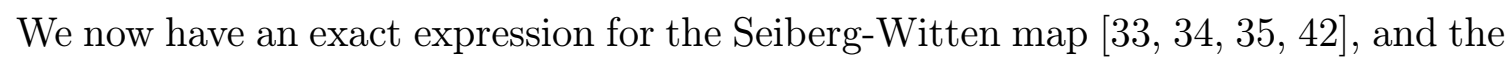
D-particle density $J_{D 0}(p)$ is given by the open Wilson line $\left(\bar{A}_{-} \overline{1} \overline{6}_{1}^{\prime}\right)$. It would therefore be important to study correlation functions of the open Wilson line in NCCS theory.

Another open problem is how to describe the hierarchy of different filling fractions in the brane picture. This hierarchy was explained in [i] $\overline{4} \overline{3}]$ by an iterative procedure of quantizing a gas of quasiparticles or quasiholes of a fluid with a given filling fraction, to produce a fluid with a different filling fraction. The result is

$$
\nu=\frac{1}{k+\frac{\alpha_{1}}{p_{1}+\frac{\alpha_{2}}{p_{2}+\ddots}}},
$$

where $k=1,3,5, \ldots, p_{i}=2,4,6, \ldots$, and $\alpha_{i}= \pm 1$, depending on whether one uses quasiparticles or quasiholes. For example, the $\nu=2 / 3$ state is obtained from the $\nu=1$ state by choosing $\alpha_{1}=1$ and $p_{1}=2$, and the $\nu=2 / 5$ state is obtained from the $\nu=1 / 3$ state with $\alpha_{1}=-1$ and $p_{1}=2$.

We have seen that a filling fraction $n / k$ fluid can be described by a configuration of $n$ D2-branes and $2 k$ D8-branes in a background $B$ field. Let us propose a possible scenario which accommodates the above hierarchy structure. Consider the case with $\nu=2 / 5$ by starting with $\nu=1 / 3$. Since $1 / 3=5 / 15$, we can also realize $\nu=1 / 3$ by $2 \times 15$ D8-branes and 5 D2-branes. The world-volume theory is U(5) NCCS theory at level $k=15$. If we increase the filling fraction by decreasing $G_{2}$, while keeping the total D-particle charge fixed, quasiparticles will appear. Since quasiparticles correspond to the ends of strings on the D2-branes, each carries a charge which is $1 / 15$ the unit D-particle charge. However, in order to make a U(5) invariant state one must combine five strings ending on the five different D2-branes. This U(5) singlet quasiparticle then actually has a charge $5 / 15=1 / 3$. This is the true quasiparticle 
of the $\nu=1 / 3$ state. As we continue to decrease $G_{2}$ more and more quasiparticles are produced, until the filling fraction reaches the value $2 / 5$. Here we notice that

$$
\frac{2}{5}=\frac{1}{3}+\frac{1}{15}=\frac{5}{15}+\frac{1}{15}=\frac{6}{15} \text {. }
$$

We therefore propose that at this point the quasiparticles condense, much like the original D-particles, to form another D2-brane, giving a total of $n=6$, and thus $\nu=6 / 15=2 / 5$. It is straightforward to extend this scenario down the hierarchy by starting with more D8-branes. To argue that this is more than an amusing scenario, however, requires a better understanding of the dynamics of quasiparticles and quasiholes. In the matrix-model picture, construction of multi-centered solutions of quasiparticles and quasiholes, and the identification of their collective coordinates would also be important.

\section{Acknowledgments}

Y.O. would like to thank Koji Hashimoto, Shinji Hirano, Ikuo Ichinose and Hirosi Ooguri for useful discussions. J.B. would like to thank Simeon Hellerman, Lenny Susskind, and Mark van Raamsdonk for helpful discussions. The work of O.B. was supported in part by the DOE under grant no. DE-FG03-92-ER40701, and by a Sherman Fairchild Prize Fellowship. The research of Y.O. was supported in part by the DOE grant DE-FG03-92ER40701. The work of J.B. was supported in part by the DOE grant DE-AC03-76SF00515.

\section{References}

[1] E. Fradkin, Field theories of condensed matter systems, Redwood City, USA: AddisonWesley (1991) 350 p. (Frontiers in physics, 82).

[2] S. M. Girvin The Quantum Hall Effect, R.E. Prange ed., Springer-Verlag, New-York, 1986.

[3] P. A. Lee and N. Nagaosa, Gauge theory of the normal state of high-Tc superconductors, 'Phys. Rev. B $\mathbf{4} 6$ (1992) 5621

[4] L. Susskind, The quantum Hall fluid and non-commutative chern simons theory, hep-th/0101029.

[5] S. Bahcall and L. Susskind, Fluid dynamics, Chern-Simons theory and the quantum Hall effect, 'Int. J. Mod. Phys. B $\overline{5}(19 \overline{9} \overline{1})-\overline{2} \overline{3} \overline{\mathrm{k}}$

[6] S. Hellerman and M. Van Raamsdonk, Quantum Hall physics equals noncommutative field theory, 'J. 
[7] R.B. Laughlin, Anomalous quantum Hall effect: an incompressible quantum fluid with

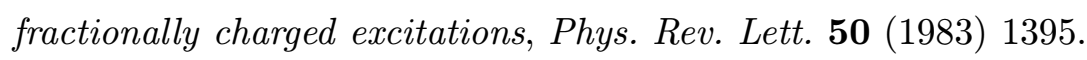

[8] D. Karabali and B. Sakita, Chern-Simons matrix model: coherent states and relation to laughlin wavefunctions, hep-th/0106016!.

[9] A. Giveon and D. Kutasov, Brane dynamics and gauge theory, Rev. Mod. Phys.

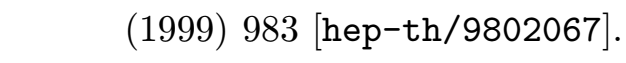

[10] A. Connes, M.R. Douglas and A. Schwarz, Noncommutative geometry and matrix theory: compactification on tori, 'U. High Energy Phys 02 (1998) 003 [hep-th/9711162].

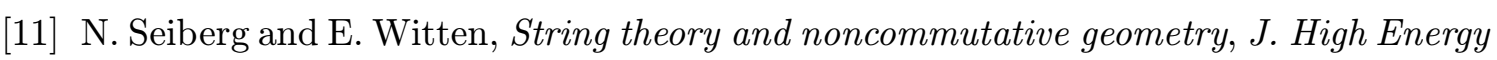

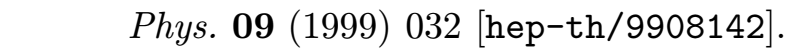

[12] M.R. Douglas and N.A. Nekrasov, Noncommutative field theory, hep-th/0106048.

[13] L.J. Romans, Massive $N=2 A$ supergravity in ten-dimensions, PPhys. Lett. (1)

[14] E. Bergshoeff, M. de Roo, M.B. Green, G. Papadopoulos and P.K. Townsend, Duality of type II 7-branes and 8-branes, iNucl. Phys. B 470 1996$) 113$ lhep-th/9601150il.

[15] T. Banks, W. Fischler, S.H. Shenker and L. Susskind, $M$ theory as a matrix model: a

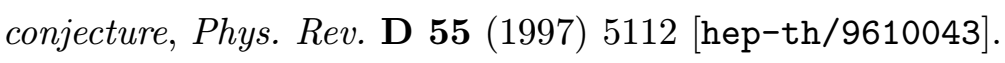

[16] N. Ishibashi, H. Kawai, Y. Kitazawa and A. Tsuchiya, A large- $N$ reduced model as superstring, Nucl. Phys. B 498 (1997) 467; thep-th/961211

[17] N. Seiberg, A note on background independence in noncommutative gauge theories, matrix model and tachyon condensation, ij. High Energy Phys. 09 $(2000))_{0} 03_{1}^{\prime}$ [hep-th/0008013.

[18] J.H. Brodie, L. Susskind and N. Toumbas, How bob laughlin tamed the giant graviton

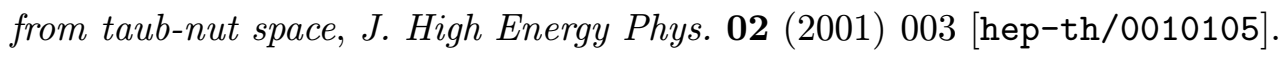

[19] A.P. Polychronakos, Quantum Hall states as matrix Chern-Simons theory, 'ST. $\bar{H}^{-} \overline{i g h}$

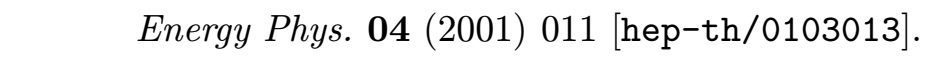

[20] S. Hellerman and L. Susskind, Realizing the quantum Hall system in string theory, hep-th/0107200!

[21] J. Polchinski, String theory, vol. 2: superstring theory and beyond, Cambridge University Press, 1998.

[22] L. Alvarez-Gaumé and E. Witten, Gravitational anomalies, 'Nučcl. P̄hyss. (1)

[23] A.N. Redlich, Parity violation and gauge noninvariance of the effective gauge field action in three-dimensions, 'Phys. Rev. D 29 (1984) 2366 . 
[24] C.-S. Chu, Induced Chern-Simons and WZW action in noncommutative spacetime, iNucl. Phys. B 580 (2000) 352: [nep-th/0003007i].

[25] J. Polchinski and A. Strominger, New vacua for type-II string theory, 'P $2 \bar{h} y s$. :

[26] O. Bergman, M.R. Gaberdiel and G. Lifschytz, Branes, orientifolds and the creation of elementary strings, Nincl. Phys. B 509 (1998) 194, [hep-th/9705130i].

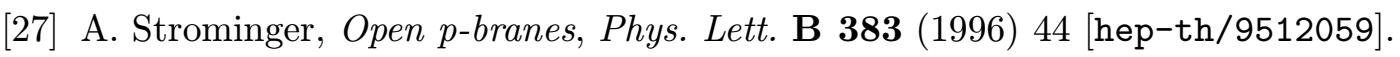

[28] U. Danielsson, G. Ferretti and I.R. Klebanov, Creation of fundamental strings by crossing D-branes, 'Phys. Rev. Lett. 79 (1997) 1984, thep-th/9705084i.

[29] T. Banks, N. Seiberg and E. Silverstein, Zero and one-dimensional probes with $N=8$

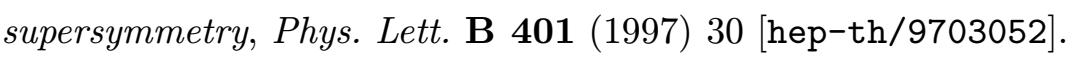

[30] I. Taylor, Washington and M. Van Raamsdonk, Multiple D0-branes in weakly curved backgrounds, iNucl. Phys. B 558 (1999) 63 i hep-th/9904095'.

[31] I. Taylor, Washington and M. Van Raamsdonk, Multiple Dp-branes in weak background fields, iNucl. Phys. B 573 (2000) 703 i⿺辶-

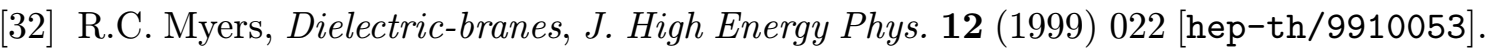

[33] Y. Okawa and H. Ooguri, An exact solution to Seiberg-Witten equation of noncommu-

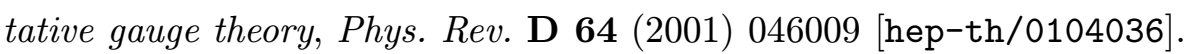

[34] S. Mukhi and N.V. Suryanarayana, Gauge-invariant couplings of noncommutative branes to Ramond-Ramond backgrounds, iJ. High Energy Phys. 05 [hep-th/0104045i.

[35] H. Liu and J. Michelson, Ramond-Ramond couplings of noncommutative D-branes,

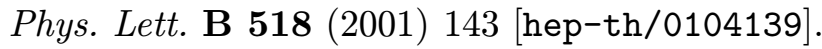

[36] S.R. Das and S.-J. Rey, Open wilson lines in noncommutative gauge theory

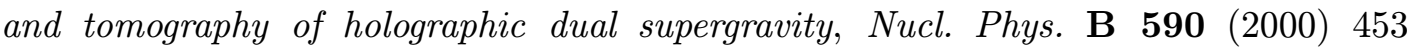
[hep-th/0008042in.

[37] N. Ishibashi, S. Iso, H. Kawai and Y. Kitazawa, Wilson loops in noncommutative Yang-Mills, Nيucl. Phys. B 573 (2000) 573 inep-th/9910004in.

[38] D.J. Gross, A. Hashimoto and N. Itzhaki, Observables of non-commutative gauge theories, iAdv. Theor. Math. Phys. 4 (2000) 893 [hep-th/0008075].

[39] K. Hashimoto and H. Ooguri, Seiberg-Witten transforms of noncommutative solitons,

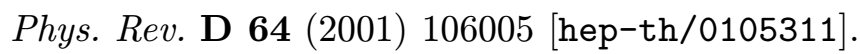

[40] Y. Okawa and H. Ooguri, How noncommutative gauge theories couple to gravity, iNoucl:i

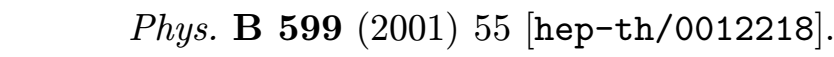




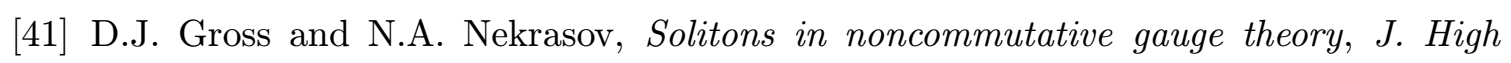

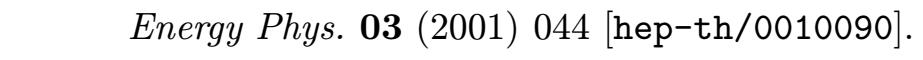

[42] H. Liu, *-Trek II: *n operations, open Wilson lines and the Seiberg-Witten map, iNüucl.: :

[43] F.D.M. Haldane, Fractional quantization of the hall effect: a hierarchy of incompress-

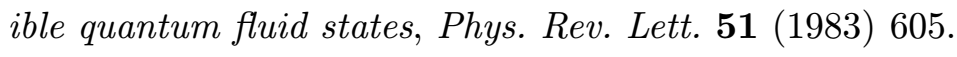

